\title{
Relationship between prospective memory and vigilance: Evidence from ERP
}

\author{
WANG Ya ${ }^{1}$, LI XueBing ${ }^{1}$, HUANG Jia ${ }^{1}$, CAO XiaoYan ${ }^{1,2}$, CUI JiFang ${ }^{3}$, ZHAO Qing ${ }^{1,2}$, \\ WANG YuNa ${ }^{1,2}$, SHUM David $\mathrm{H} \mathrm{K}^{4} \&$ CHAN Raymond $\mathrm{C} \mathrm{K}^{1 *}$ \\ ${ }^{1}$ Neuropsychology and Applied Cognitive Neuroscience Laboratory, Key Laboratory of Mental Health, Institute of Psychology, Chinese Academy \\ of Sciences, Beijing 100101, China; \\ ${ }^{2}$ Graduate University of Chinese Academy of Sciences, Beijing 100049, China; \\ ${ }^{3}$ National Institute of Education Sciences, Beijing 100088, China; \\ ${ }^{4}$ Behavioural Basis of Health Program, Griffith Health Institute and School of Applied Psychology, Griffith University, Brisbane 4122, Australia
}

Received February 3, 2012; accepted April 26, 2012; published online August 16, 2012

\begin{abstract}
Event-related potentials (ERPs) were used in this study to investigate the neural correlates of prospective memory (PM) and vigilance. Twenty college or graduate students participated in this study. They were administered a PM and a vigilance task and physiological data were collected at the same time. Behavioral results showed that the RT associated with PM cues was longer than those associated with vigilance targets. ERP results showed that PM cues and vigilance targets did not show significant difference in the N2 but PM cues evoked greater N300 than vigilance targets, and vigilance targets evoked greater parietal positivity/P3 than PM cues, suggesting vigilance and PM have similar but also distinctive neural basis.
\end{abstract}

prospective memory, vigilance, ERP

Citation: Wang Y, Li X B, Huang J, et al. Relationship between prospective memory and vigilance: Evidence from ERP. Chin Sci Bull, 2012, 57: 4057-4063, doi: $10.1007 / \mathrm{s} 11434-012-5306-9$

Prospective memory (PM) refers to remember to do something at a particular situation in the future [1]. It is important for everyday living, for example, remembering to post a letter on the way home from work. PM is characterized by several features: (1) low frequency of occurrence, that is, the PM cue occurs relatively infrequently within ongoing trials; (2) a delay between intention formation and realization, that is, the intention could not be executed immediately after it was formed; (3) attention is directed to another task, that is, during the delayed period, the individual is busy doing another task - the ongoing task; and (4) there is no external reminder for execution of the remembered action, that is, the individual has to self-initiate the execution of the intention [2]. There are five stages of cognitive process underlying PM performance, namely, intention formation, intention maintenance, performance interval and

*Corresponding author (email: rckchan@psych.ac.cn) cue detection, initiation and execution of intention, and evaluation of outcome [3].

Vigilance or sustained attention is defined as the ability to maintain attention for prolonged periods of time [4]. A vigilance task usually requires someone to detect infrequent targets among frequent non-targets. There are several similar characteristics between PM and vigilance tasks. First, a vigilance component is always involved in PM task [5,6]. Second, PM is similar to vigilance in that participants are usually required to monitor a continuous series of stimuli to detect the appearance of a low frequency target and to respond when a target is detected [7]. However, there are also some unique features in a PM task that distinguish it from a vigilance task. For example, whereas the emphasis of a PM task is the ongoing activity, the detection of targets is the emphasis of a vigilance task [7,8]. The retrieval processes underlying PM and vigilance are also different. Brandimonte and colleagues [6,9] suggest that in a PM task, 
monitoring is not a continuous process during the maintenance interval. Participants undertaking an event-based PM task do not always check their intention. Instead, they just wait for the appearance of the cue. However, monitoring is continuous in a typical vigilance task. Brandimonte et al. [6] also found that reaction time (RT) in vigilance was longer than that in PM. They, therefore, suggested that vigilance relies more on continuous monitoring for the targets but PM relies more on spontaneous retrieva.

There are a growing number of studies that examined the neural basis of PM. Recent studies suggest that PM is specifically associated with the prefrontal lobe, particularly BA 10 [10-12]. Event-related potential (ERP) studies also suggest that there are some modulations specifically related to PM, namely, N300 and prospective positivity [13]. N300 occurs during 300-400 ms after stimulus onset at the occipital-parietal region and is related to PM cue detection. Prospective positivity occurs during 400-1200 ms after stimulus onset at the central, parietal and occipital regions and is related to intention retrieval [13]. West and his colleagues [14-18] also found that these modulations are specifically related to PM, and could be differentiated from retrospective and working memory. For example, N300 could be dissociated with N2pc elicited by targets of a target selection task [16], prospective positivity could be dissociated with P3 elicited by working memory and retrospective memory [14,19].

The typical vigilance task used in ERP is the oddball paradigm that requires participants to attend to a rare target among more frequent non-targets. In this kind of task, targets results in N2, P3 and a slow wave. The peak amplitude of P3 is found in the parietal area, and the medial temporal lobe and frontal lobe may also be involved [20]. While N2 indicates visual target detection, P3 reflects updating of target information or context in working memory or categorization of stimulus [21]. Studies found that P3 is sensitive to frequency of target, task relevance and task difficulty $[22,23]$ but not sensory modalities, that is, both auditory and visual stimuli would evoke P3 [24].

West et al. [7] compared the neural correlates of PM and vigilance, and found some differences in modulations evoked by PM and vigilance. For example, the ascending arm (400-600 ms) of parietal positivity evoked by PM and the late positive complex (LPC) evoked by the oddball task were different. This was because of the increase in amplitude associated with ongoing activity trials during this epoch in PM relative to the oddball task. Also for the 600-800 ms epoch of the oddball task, this arm of LPC showed significant differences between all trial types: target, lure (stimuli that had part but not all features of the target), and nontarget trials. However, for the PM task, there was greater positivity for PM cue trials than PM lure or ongoing trials (the latter two did not show significant difference) and frontal slow wave elicited by PM was attenuated in the oddball task. Nevertheless, these results are based on a between experiment comparison and they emphasized the role of PM lure in PM and vigilance task. Thus, it would be better to undertake a within-subject comparison and make a more direct comparison between PM and vigilance.

Given the above findings, the present study aimed to compare behavioural performance and ERP modulations between PM and vigilance. Since the PM task is a dual task paradigm while the vigilance is a single task, we hypothesized that the RT of ongoing trials in a PM task would be longer than the RT of non-target trials in a vigilance task. For ERP results, since PM has two cue detection processes (ongoing task cue detection as the primary task and PM cue detection as the secondary task), and vigilance has only one target detection process, we hypothesized that the PM and vigilance would have a similar $\mathrm{N} 2$ component and $\mathrm{PM}$ would have a specific N300 component. Moreover, since the PM task is a dual task and attention resources allocated to PM cues was less than that allocated to vigilance targets in the vigilance task, we hypothesized that the prospective positivity evoked by PM cues would have smaller amplitude than the P3 evoked by vigilance targets. Finally, since the PM task is more difficult than the vigilance task, we hypothesized that the prospective positivity evoked by PM cues would have a longer latency than the P3 evoked by vigilance targets. By comparing $\mathrm{PM}$ and vigilance, we hoped to clarify the processing mechanisms of PM.

\section{Method}

\subsection{Participants}

Twenty undergraduate or graduate students (12 males and eight females) from three universities in Beijing took part in the study. All participants were right-handed and had normal or corrected to normal vision. None had a history of neurological or psychiatric disease. Mean age and mean number of year of education were 22.85 years $(\mathrm{SD}=1.50)$ and 16.25 years $(\mathrm{SD}=2.92)$, respectively. All of them were paid for their participation.

\subsection{Materials and tasks}

(i) PM task. The PM paradigm of the study was developed based on the task used by West et al. [7]. The stimuli of the PM task were two arrows between two bars presented in the centre of the screen. The bars were either black or white in colour, and the arrows were one black and one white pointing to different directions (one directed to left and one directed to right) (Figure 1(a)). The participants were asked to judge the direction of the black arrows (the ongoing task) and were instructed to press the "J" key on the keyboard if the black arrow pointed to the right (45\% of the trials) and to press the "F" key if it pointed to the left ( $45 \%$ of the trials). Occasionally ( $10 \%$ of the trials) the two arrows would point to the same direction (both arrows point to left or right). In that case, participants were asked to press 


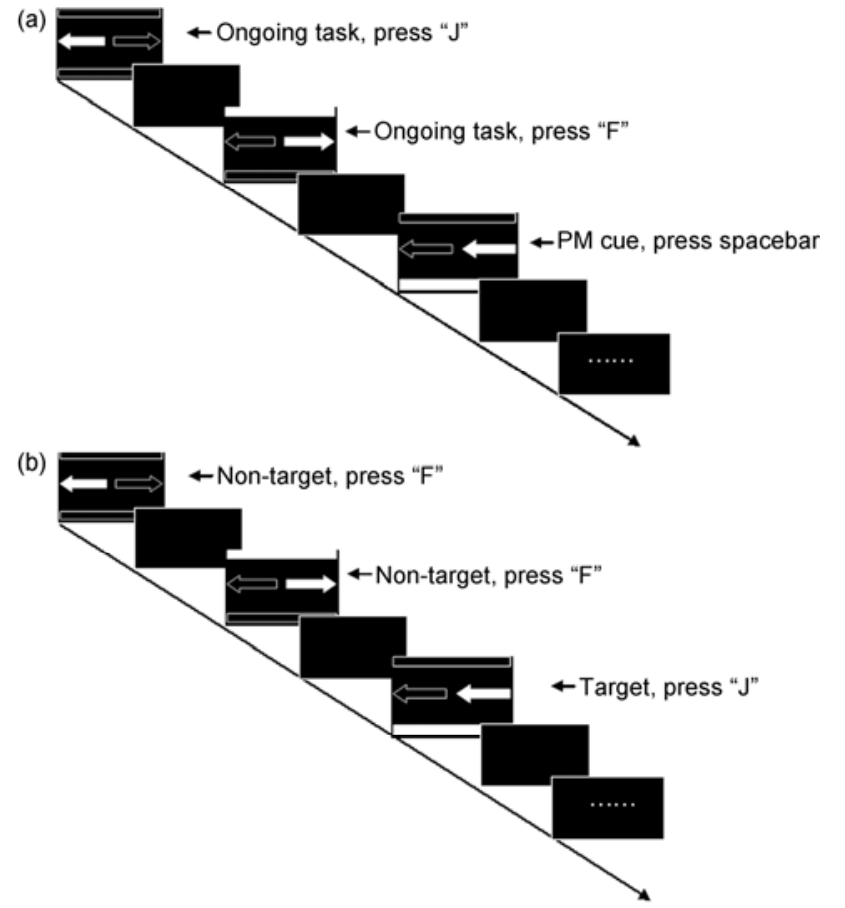

Figure 1 (a) Illustration of the PM task. (b) Illustration of the vigilance task.

the spacebar (the PM task). Each pair of stimuli appeared on the screen for $300 \mathrm{~ms}$, then the screen would go blank for 1800-2000 ms randomly, and then the next pair of stimuli would appear. There were 120 trials in each block, and a total of five blocks were presented. Participants were given a short break between blocks.

(ii) Vigilance task. For the vigilance task, the stimuli were the same as the PM task. However, participants were only asked to judge whether the two arrows were in the same direction or not. If the arrows were in the same direction (target, $10 \%$ of trials), the participants were instructed to press the " $\mathrm{J}$ " key; if the arrows were not in the same direction (non-target, $90 \%$ of trials), the participants were instructed to press the "F" key (for illustration, see Figure 1(b)).

\subsection{Procedure}

This study was approved by the ethics committee of the Institute of Psychology, Chinese Academy of Sciences. All participants signed an informed consent form before the study. The participants were given a practice block before the actual PM and vigilance blocks. The practice block included 20 trials, 18 of which were ongoing task or nontarget trials, 2 of which were PM or target trials. The PM and vigilance tasks were completed in a counterbalanced order across participants.

\subsection{EEG recording and analysis}

EEG with bandpass of $0.05-100 \mathrm{~Hz}$ and sampling rate of
$1000 \mathrm{~Hz}$ was recorded from 64 scalp sites using $\mathrm{Ag} / \mathrm{AgCl}$ electrodes (NeuroScan Inc., Herndon, Virgina, USA), using the left and right mastoids as references. Horizontal electrooculogram (EOG) recording electrodes were positioned at the outer canthi of both eyes, and vertical EOG recording electrodes were positioned above and below the left eye. The impedances of all electrodes were kept below 5 $\mathrm{k} \Omega$ during the experiment.

A $30 \mathrm{~Hz}$ low-pass filter was used for offline digital filter with the EEG and EOG data. The EEG data were epoched from $200 \mathrm{~ms}$ before stimuli onset to $1000 \mathrm{~ms}$ after stimuli onset. Trials with artifacts larger than $\pm 50 \mu \mathrm{V}$ were rejected. Only the trials with correct responses were analyzed in the ERP data analysis.

Statistical analyses included the following 25 electrodes: PO7, O1, OZ, O2, and PO8 for the occipital region; P3, P4, $\mathrm{P} 7, \mathrm{P} 8$, and PZ for the parietal region; T7, C3, CZ, C4, and T8 for the central region; FC1, FC2, FT7, FT8, and FZ for the frontal-central region; FP1, FP2, F7, F8, and FPZ for the frontal-polar region.

\section{Results}

\subsection{Behavioural data}

Participants' performance on the PM ongoing task and their detection of vigilance non-targets were nearly perfect. Participants' response to the PM cue and their detection of vigilance targets showed similar accuracy (Table 1). Paired sample $t$-tests revealed that there was no significant difference between PM ongoing task accuracy and vigilance non-target accuracy, $t(19)=-0.40, P=0.694$. In addition, PM ongoing task RT and vigilance non-target RT were not found to be significantly different from each other, $t(19)=$ $1.14, P=0.269$. PM cue accuracy and vigilance target accuracy were not found to be significantly different, $t(19)=$ $-0.95, P=0.353$. However, the RT of PM cue was found to be significantly longer than the vigilance target RT, $t(19)=$ 4.00, $P=0.001$.

\subsection{EEG data}

The grand average ERP data for PM cue trials, ongoing

Table 1 Behavioural data of PM and vigilance task ${ }^{\text {a) }}$

\begin{tabular}{lrrrr}
\hline & Min & Max & Mean & SD \\
\hline PM ongoing accuracy & 0.99 & 1.00 & 0.99 & 0.01 \\
PM ongoing RT (ms) & 474.51 & 832.23 & 586.56 & 91.02 \\
PM cue accuracy & 0.70 & 0.98 & 0.84 & 0.09 \\
PM cue RT (ms) & 612.66 & 1038.18 & 751.32 & 100.99 \\
VIGI non-target accuracy & 0.99 & 1.00 & 0.99 & 0.01 \\
VIGI non-target RT (ms) & 421.33 & 880.70 & 566.97 & 134.12 \\
VIGI target accuracy & 0.58 & 1.00 & 0.85 & 0.10 \\
VIGI target RT (ms) & 547.92 & 880.73 & 684.88 & 112.55 \\
\hline
\end{tabular}

a) PM, prospective memory; VIGI, vigilance; RT, reaction time. 


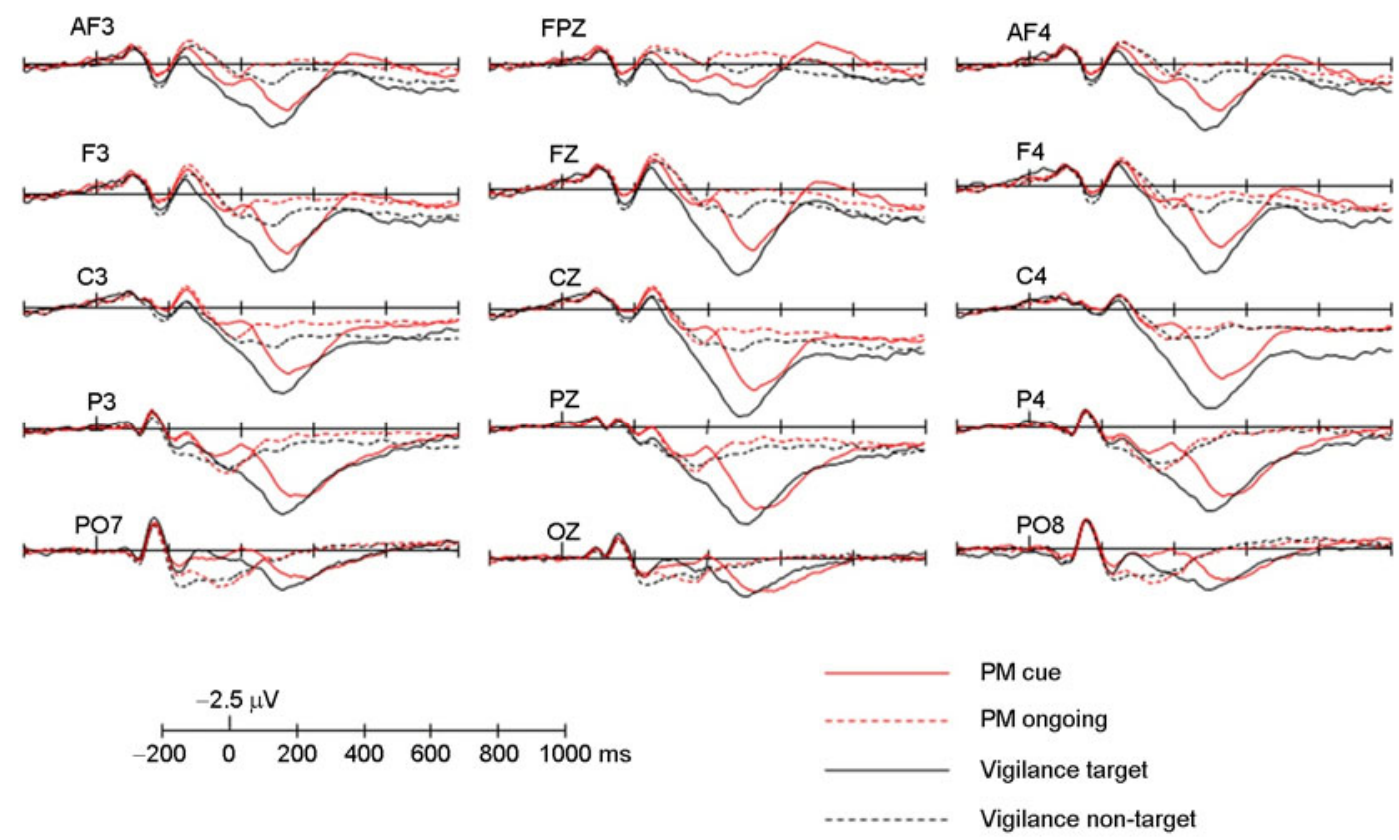

Figure 2 Grand average ERPs for PM cue, PM ongoing task, vigilance target and vigilance non-target.

tasktrials, vigilance targets, and vigilance non-targets at selected electrodes are presented in Figure 2. The N2, N300, parietal positivity for PM cue were observed, and N2, P300 for vigilance targets were observed. We select 200-300 ms interval to capture the trial effects on $\mathrm{N} 2$, and $350-450 \mathrm{~ms}$ interval to capture N300. These effects mainly occurred in the frontal, central, parietal and occipital regions and reflected the cue-detection processing. The $450-600 \mathrm{~ms}$ intervals indicated the parietal positivity or P3. Activities during the 450-600 ms interval reflected intention retrieval and categorization that mainly occurred in the parietal, central and frontal regions.

To examine the similarity and differences between PM and vigilance, we compared the PM cues and vigilance targets. Since the modulations of ongoing trials in PM task and non-targets in vigilance task showed a similar pattern, we compared the waves evoked by PM cues and vigilance targets instead of comparing the difference wave between PM cue and PM ongoing trials to the difference wave between vigilance target and vigilance non-target. Figure 3 showed the topographic maps of PM cue and vigilance target. A series of 2 (Trial: PM cue, vigilance target) $\times 5$ (Region: frontal-polar (F7, FP1, FPZ, FP2, F8), frontal-central (FT7, FC1, FZ, FC2, FT8), central (T7, C3, CZ, C4, T8), parietal (P7, P3, PZ, P4, P8), and occipital (PO7, O1, OZ, O2, PO8)) $\times 5$ (Electrode) ANOVAs reflecting mean amplitude between 200-300 ms (N2), 350-450 ms (N300), and 450-600 $\mathrm{ms}$ (prospective positivity/P3) and latency of prospective positivity/P3. Only the main effects and interactions involving Trial were presented in the results. GreenhouseGeisser correction was used for all analysis. During the 200-300 ms interval, difference between PM cues and

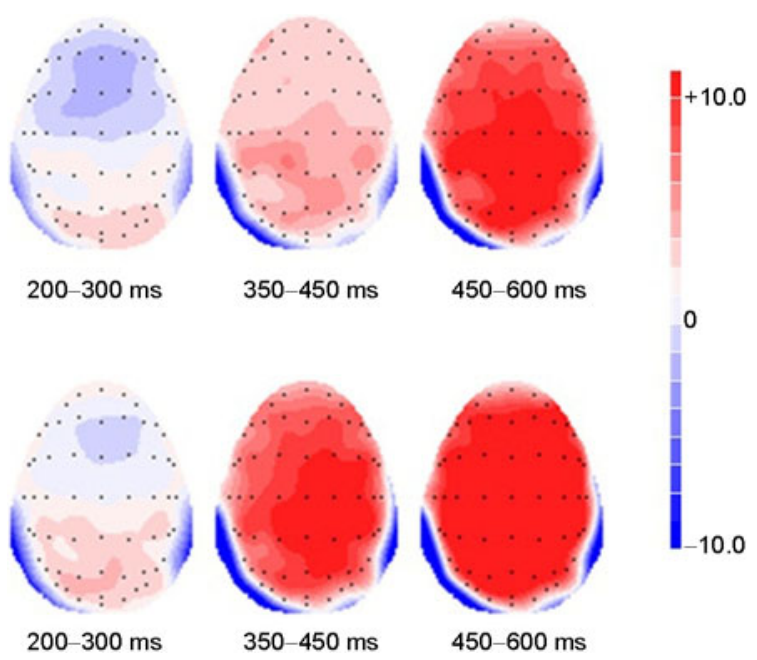

Figure 3 Topographical maps of the voltage amplitudes $(\mu \mathrm{V})$ for PM cue and vigilance target. The upper three maps indicate PM cue, the lower three maps indicate vigilance target.

vigilance targets (effect of Trial) was non-significant, $F(1,19)=3.30, P>0.05$. Interaction of Trial $\times$ Electrode was significant, $F(4,76)=4.08, P=0.021$.

During the $350-450 \mathrm{~ms}$ interval, PM cues evoked greater negative waves than vigilance targets, $F(1,19)=21.71, P<$ 0.001 . The interaction of Trial $\times$ Region, $F(4,76)=10.53, P$ $<0.001$, Trial $\times$ Electrode, $F(4,76)=14.22, P<0.001$, and Trial $\times$ Region $\times$ Electrode, $F(16,304)=20.87, P<0.001$, were significant. Trial $\times$ Region interaction was analyzed further in a series of post hoc analysis that contrast the effect of Trial in each region. Table 2 showed the statistics of the analysis. The effect of Trial was significant in all the 
occipital, parietal, central, frontal-central and frontal-polar regions. The Trial $\times$ Electrode interaction was significant in the parietal, central and frontal-central regions. Further analysis showed that trial difference was significant in all electrodes, with difference greater in midline electrodes than right lateral electrodes which in turn greater than left lateral electrodes.

During the 450-600 ms interval, vigilance targets evoked greater positivity than PM cues, $F(1,19)=18.81, P<0.001$. The interactions of Trial $\times$ Region, $F(4,76)=5.49, P=$ 0.017 , Trial $\times$ Electrode, $F(4,76)=3.96, P=0.023$, and Trial $\times$ Region $\times$ Electrode, $F(16,304)=9.07, P<0.001$, were significant. Post hoc analysis results of the Trial $\times$ Region interaction were presented in Table 2. Further analyses showed that the effect of Trial was significant in the parietal, central and frontal-central regions. The Trial $\times$ Electrode interaction was significant in the occipital, parietal, central, and frontal-central regions. Follow up analyses showed that in the occipital region, the trial difference was significant at electrode PO7; in the parietal, central and frontal-central regions, the trial difference was significant in all electrodes, with greater differences observed in the midline and lateral electrodes. We also analyzed the latency of this component and the results revealed that the latency of PM cues was larger than that of vigilance targets, $F(1,19)=17.39, P<$ 0.001. All interactions involving Trial were non-significant.

Table 2 Comparison of amplitude of PM cue and vigilance target

\begin{tabular}{|c|c|c|c|}
\hline & \multirow{2}{*}{ Effect } & \multicolumn{2}{|c|}{ PM cue vs vigilance target } \\
\hline & & $F$ & $P$ \\
\hline \multicolumn{4}{|l|}{ Part a: $350-450 \mathrm{~ms}$} \\
\hline \multirow[t]{2}{*}{ Frontal-polar } & Trial & 5.72 & 0.027 \\
\hline & Trial $\times$ Electrode & 1.15 & 0.334 \\
\hline \multirow[t]{2}{*}{ Frontal-central } & Trial & 20.23 & $<0.001$ \\
\hline & Trial $\times$ Electrode & 21.48 & $<0.001$ \\
\hline \multirow[t]{2}{*}{ Central } & Trial & 27.05 & $<0.001$ \\
\hline & Trial $\times$ Electrode & 26.84 & $<0.001$ \\
\hline \multirow[t]{2}{*}{ Parietal } & Trial & 32.82 & $<0.001$ \\
\hline & Trial $\times$ Electrode & 13.86 & $<0.001$ \\
\hline \multirow[t]{2}{*}{ Occipital } & Trial & 17.36 & $<0.001$ \\
\hline & Trial $\times$ Electrode & 2.50 & 0.078 \\
\hline \multicolumn{4}{|l|}{ Part b: $450-600 \mathrm{~ms}$} \\
\hline \multirow[t]{2}{*}{ Frontal-polar } & Trial & 3.62 & 0.072 \\
\hline & Trial $\times$ Electrode & 0.73 & 0.490 \\
\hline \multirow[t]{2}{*}{ Frontal-central } & Trial & 20.53 & $<0.001$ \\
\hline & Trial×Electrode & 8.52 & 0.001 \\
\hline \multirow[t]{2}{*}{ Central } & Trial & 27.01 & $<0.001$ \\
\hline & Trial $\times$ Electrode & 11.10 & $<0.001$ \\
\hline \multirow[t]{2}{*}{ Parietal } & Trial & 17.27 & 0.001 \\
\hline & Trial $\times$ Electrode & 3.32 & 0.028 \\
\hline \multirow[t]{2}{*}{ Occipital } & Trial & 2.52 & 0.129 \\
\hline & Trial×Electrode & 4.17 & 0.020 \\
\hline
\end{tabular}

\section{Discussion}

The current study showed that: (1) for behavioural results, accuracy and RT for the PM ongoing task and accuracy for the detection of the vigilance non-targets did not show significant difference, but PM cues were found to have longer RT than vigilance targets, inconsistent with our hypothesis; (2) for ERP results, PM cues and vigilance targets did not show significant amplitude difference for N2 (200-300 ms), consistent with our hypothesis; (3) PM cues evoked greater N300 than vigilance targets, consistent with our hypothesis; and (4) vigilance targets evoked greater positivity and shorter latency than PM cues, consistent with our hypothesis.

As to behavioural results, the PM ongoing and vigilance non-target trials did not show significant differences in either accuracy or RT. The accuracy of PM cues and vigilance target trials did not show significant difference either. However, contrary to the results of the present study, Brandimonte et al. [6] found that their participants had longer RTs for the non-target trials of the vigilance task than those for the ongoing trials of the PM task. The present study may not be comparable to Brandimonte et al.'s [6] study, because in their study, Brandimote et al. used a stop signal paradigm and participants were instructed to withhold their responses upon detecting the vigilance target or PM cue. The participants in the present study, on the other hand, were instructed to respond to every trial in the present study. The results of the present study suggest that a dual task PM paradigm was more difficult and needed more cognitive resources than a vigilance task. Behavioral results of the present study were also not consistent with those reported by West et al. [7]. While the oddball task (accuracy ranged from 0.94 to 1 ) was found by West et al. to have a higher accuracy than the PM task (ranged from 0.89 to 0.92 ), the accuracy for PM $(M=0.84)$ and vigilance $(M=0.85)$ were found to be similar in the present study. This maybe because in West et al.'s study, the participants only needed to respond to the targets when undertaking the vigilance task, thus it was simpler and had a higher accuracy than the PM task. However, in the present study the vigilance task was more comparable to the PM task in that the participants needed to respond to every stimuli, thus the accuracy of the PM cue trials and vigilance target trials were not significantly different. However, although the RT of ongoing activity in PM task and non-target in vigilance task were not significantly different, the RT of PM cues was significantly longer than vigilance targets, and it was consistent with the findings reported by West et al. [7].

As to the ERP results, the amplitudes of negativity during 200-300 ms between PM cues and vigilance targets did not show significant difference. Sponheim et al. [21] suggested that in a vigilance task, the $\mathrm{N} 2$ reflects the target detection process. In the present vigilance task, the N2 might indicate the detection of vigilance target, while in the PM task, the N2 might indicate the detection of target of ongo- 
ing task (the direction of black arrow). Both of these processes represent the primary activity of each task, thus evoked similar N2. This indicated that the vigilance and PM shared a similar cue detection process. PM cue evoked greater N300 (350-450 ms) than vigilance target. According to West and his colleagues $[7,13]$, this negativity is indicative of the cue detection process of PM cue. The PM cue occurs occasionally and may have represented the secondary task. For the vigilance task, vigilance targets actually did not evoke such component and this indicated that this cue detection process was specifically related to PM, consistent with the finding reported by West [13].

During 450-600 ms, vigilance targets evoked greater positivity than PM cues in the parietal, central and frontalcentral regions. According to West et al. [7], the prospective positivity was related to the intention retrieval and categorization process and had some similar characteristics as the P3. In West et al.'s [7] study, they compared the amplitudes between PM cues, and ongoing trials in a PM and a vigilance task and their results showed that for the first part of LPC (400-600 ms), the amplitude of ongoing trials increased in the PM condition compared to the vigilance condition. However, West et al.'s study was a between experiment comparison and the amplitude of vigilance targets and PM cues was not compared. In contrast, the present study provided a within-subject comparison of PM and vigilance and no significant differences were found between ongoing trials and non-target trials. In addition, the vigilance targets evoked greater positivity than PM cues. The fact that the amplitude of modulation evoked by PM cues was found to be less than that of vigilance targets indicated that the categorization process of the PM task was more difficult than that of the vigilance task. In Kok's [23] review, he suggested that in the easy condition, the two components (P3a and $\mathrm{P} 3 \mathrm{~b}$ ) added and generated greater amplitude, but in more difficult conditions, the mutual enhancement diminished, and led to a reduction in P3 amplitude. For the present results, in the PM condition, there was a small positivity followed by a larger positivity. These two modulations were separated and did not add together, whereas in the vigilance condition, there was only one large positivity (P3a and P3b overlapped and added together), and the amplitude of which was larger than in PM condition. This was also consistent with the conception that attention resources allocated to PM cue in the PM task was less than that allocated to targets in the vigilance task, and that the amplitude of prospective positivity in the PM task showed a smaller amplitude than P3 in the vigilance task. For the latency of this component, PM cue had a longer latency than vigilance targets and this was because a difficult task would lead to a longer latency of P3 [23].

This study has several limitations. First, it did not include a baseline condition where no PM has been introduced. With this condition, we could infer whether PM was automatically retrieved or strategically retrieved and be able to provide support for one PM theory or the other. Second, the responses participants need to make in PM task and vigilance task were different. While PM had three response keys, vigilance had only two response keys. This may explain some of the ERP difference. Further study is needed to adopt more comparable tasks to make comparisons.

In conclusion, the present study found that PM and vigilance may have one similar cue-detection process, but PM had another specific cue detection process, given the differences in categorization and intention retrieval processes between PM and vigilance. These findings indicate that PM and vigilance may have some common as well as unique neural correlates.

This work was supported by the Young Investigator Scientific Fund of Institute of Psychology, Chinese Academy of Sciences (O9CX073007), the National Key Technologies R\&D Program (2012BAI36B01), the National Natural Science Foundation of China (30900403, 91132701, 30770723, 81088001), Youth Innovation Promotion Association Funding of Chinese Academy of Sciences (Y1CX131003), Key Laboratory of Mental Health, Institute of Psychology, Chinese Academy of Sciences and the Knowledge Innovation Project of the Chinese Academy of Sciences (KSCX2-EW-J-8). These funding agents had no further role in the study design; in the collection, analysis and interpretation of the data; in the writing of the manuscript; and in the decision to submit the paper for publication. The authors disclaim there is no conflicts of interest for this study.

1 Einstein G O, McDaniel M A. Normal aging and prospective memory. J Exp Psychol Learn Mem Cogn, 1990, 16: 717-726

2 Brandimonte M A, Einstein G O, McDaniel M A. Prospective Memory: Theory and Applications. Mahwah, New Jersey: Lawrence Erlbaum Associates, 1996

3 Ellis J A. Prospective memory or the realization of delayed intentions: A conceptual framework for research. In: Brandimonte M, Einstein G O, McDaniel M A, eds. Prospective Memory: Theory and Applications. Mahwah, New Jersey: Erlbaum, 1996. 1-22

4 Warm J S, Parasuraman R, Matthews G. Vigilance requires hard mental work and is stressful. Hum Factors, 2008, 50: 433-441

5 Maylor E A. Age-related impairment in an event-based prospective-memory task. Psychol Aging, 1996, 11: 74-78

6 Brandimonte M A, Ferrante D, Feresin C, et al. Dissociating prospective memory from vigilance processes. Psicologica, 2001, 22: 97-113

7 West R, Wymbs N, Jakubek K, et al. Effects of intention load and background context on prospective remembering: An event-related brain potential study. Psychophysiology, 2003, 40: 260-276

8 Graf P, Uttl B. Prospective memory: A new focus for research. Conscious Cogn, 2001, 10: 437-450

9 Brandimonte M A, Bisiacchi P S, Pelizzon L. Perceptually-driven memory for intentions: A study with children and adults. Cogn Technol, 2000, 5: 20-25

10 Burgess P W, Quayle A, Frith C D. Brain regions involved in prospective memory as determined by positron emission tomography. Neuropsychologia, 2001, 39: 545-555

11 Okuda J, Fujii T, Yamadori A, et al. Participation of the prefrontal cortices in prospective memory: Evidence from a PET study in humans. Neurosci Lett, 1998, 253: 127-130

12 Burgess P W, Gonen-Yaacovi G, Volle E. Functional neuroimaging studies of prospective memory: What have we learnt so far? Neuropsychologia, 2011, 49: 2246-2257

13 West R. The cognitive neuroscience of prospective memory. In: Kliegel M, McDaniel M A, Einstein G O, eds. Prospective Memory: Cognitive, Neuroscience, Developmental, and Applied Perspectives. New York: Lawrence Erlbaum Associates, 2008. 261-282

14 West R, Bowry R, Krompinger J. The effects of working memory 
demands on the neural correlates of prospective memory. Neuropsychologia, 2006, 44: 197-207

15 West R, Krompinger J. Neural correlates of prospective and retrospective memory. Neuropsychologia, 2005, 43: 418-433

16 West $\mathrm{R}, \mathrm{Wymbs} \mathrm{N}$. Is detecting prospective cues the same as selecting targets? An ERP study. Cogn Affect Behav Neurosci, 2004, 4: 354-363

17 West R. The influence of strategic monitoring on the neural correlates of prospective memory. Mem Cogn, 2007, 35: 1034-1046

18 West R, McNerney M W, Travers S. Gone but not forgotten: The effects of cancelled intentions on the neural correlates of prospective memory. Int J Psychophysiol, 2007, 64: 215-225

19 West R, Bowry R. Effects of aging and working memory demands on prospective memory. Psychophysiology, 2005, 42: 698-712

20 Riccio C A, Reynolds C R, Lowe P, et al. The continuous performance test: A window on the neural substrates for attention? Arch Clin Neuropsychol, 2002, 17: 235-272

21 Sponheim S R, McGuire K A, Stanwyck J J. Neural anomalies during sustained attention in first-degree biological relatives of schizophrenia patients. Biol Psychiatry, 2006, 60: 242-252

22 Donchin E. Surprise!... Surprise? Psychophysiology, 1981, 18: 493513

23 Kok A. On the utility of $\mathrm{P} 3$ amplitude as a measure of processing capacity. Psychophysiology, 2001, 38: 557-577

24 Romero R, Polich J. P3 (00) habituation from auditory and visual stimuli. Physiol Behav, 1996, 59: 517-522

Open Access This article is distributed under the terms of the Creative Commons Attribution License which permits any use, distribution, and reproduction in any medium, provided the original author(s) and source are credited. 